\title{
The effect of virtual reality on motor anticipation and hand function in patients with subacute stroke: A randomised trial on movement- related potential.
}

\author{
Ling Chen \\ Guangzhou University of Chinese Medicine \\ Yi Chen \\ Guangzhou University of Chinese Medicine \\ Wai Leung Ambrose Lo ( $\square$ ambroselo0726@outlook.com ) \\ https://orcid.org/0000-0001-7350-2157
}

\section{Research}

Keywords: Stroke, upper limb function, motor planning, contingent negative variation

Posted Date: March 18th, 2020

DOI: https://doi.org/10.21203/rs.2.21922/v2

License: (c) (i) This work is licensed under a Creative Commons Attribution 4.0 International License. Read Full License

Version of Record: A version of this preprint was published at Neural Plasticity on January 24th, 2022. See the published version at https://doi.org/10.1155/2022/7399995. 


\section{Abstract}

Background: Impaired cognitive ability to anticipate the required control may affect functional recovery. The cortical excitability of task related motor anticipation for upper limb movement induced by virtual reality (VR) training is unknown. Aims: To investigate the changes of motor anticipation, cortical excitability and upper limb function induced by VR training in subacute stroke survivors. Methods: Upper limb hemiparesis resulting from the first occurrence of stroke within 1 to 6 months were recruited. Participants were randomly allocated to conventional therapy or VR intervention. Electroencephalogram (EEG) and electromyography (EMG) were simultaneously recorded during palmar grasp motion. Outcome measures of contingent negative variation (CNV) latency, mean peak amplitude, electromyography (EMG) reaction time, Upper Limb Fugl-Meyer Assessment (UL-FMA) and the Action Research Arm Test (ARAT) were recorded pre and post intervention. The EEG and EMG differences between the two groups were assessed by mixed model ANOVA ( $p=0.05)$. The differences in ULFMA and ARAT were assessed Wilcoxon signed-rank test $(\mathrm{p}=0.05)$. Results: The reduction in EMG reaction time difference, CNV latency and mean peak amplitude were significantly lower in the VR group than the control group when executing movement with the paretic hand. ARAT and UL-FMA scores were significantly higher in the VR group than the control group post intervention. Conclusions: The findings indicated a reduction in brain computational demand during palmar grasp task. Improvements in clinical function in the VR group suggested that VR intervention may be more beneficial in improving upper limb function patients with subacute stroke. Improvement in upper limb function may be related to improvement of the neural anticipatory process. Trial registration: Chinese Clinical Trial Registry (Registration No.: ChiCTRIOC-15006064). Registered on 11 May 2015. Retrospectively registered. http://www.chictr.org.cn/searchprojen.aspx

\section{Introduction}

Stroke is one of the most severe issues encountered by the ageing population ${ }^{1}$ as it is among the leading causes of long-term disability worldwide ${ }^{2}$. Published epidemiological study reported that approximately $60 \%$ of stroke survivors have motor dysfunction and $40 \%$ continue to have severe disability of the upper extremity ${ }^{3}$. Statistically, only $5 \%$ to $20 \%$ of stroke survivors recovered their upper limb function completely, $25 \%$ of them recover part of upper limb function, and $60 \%$ of them lost upper limb function completely 4 . Motor dysfunction of upper extremity contribute lead to reduce ability to perform motor tasks such as reaching and grasping ${ }^{5} \llbracket$ which in turn affects the ability to perform activity of daily living such as eating, dressing and washing ${ }^{6,7}$.

The clinical outcomes of upper extremity rehabilitation is influenced by cognitive function which directly affects the ability to acquire and execute functional skills ${ }^{8,9}$. Motor anticipation is a key component of cognitive function which requires high level of brain cognitive process $^{10}$. It is one of the essential element for successful motor execution since volitional movements are preceded by a period of planning, during which parameters are set for specific upcoming movement to achieve a specific goal ${ }^{11}$. Early literature on non-human primate reported increase in neural spike rate prior to movement initiation ${ }^{12}$ which is considered as a key signature of cognitive preparatory activity 11. Study in human indicated that the brain activates 1.5 seconds before the execution of movements, while people perceive motor intention at 500 to $800 \mathrm{~ms}$ after the activation of the motor cortex ${ }^{13}$. Motor preparation requires the prediction of the physical properties of static objects and the dynamic loads generated by the objects in space ${ }^{5,14-16}$. Studies in stroke survivors indicated that the ability to anticipate the required control to execute motor task is lacking ${ }^{17}$ which contribute to motor intentionality and power accuracy out of control. Literature indicated impaired anticipatory ability in stroke patients may present clinically as a marked increased grip force during object manipulation of the upper extremity ${ }^{18-21}$.

The electrophysiological process that is associated with motor anticipation and whether changes in motor anticipation is associated with functional improvement are unclear ${ }^{22}$. Exiting studies indicated that a loss of frequency alpha band on the ipsilesional hemisphere were related to poor motor recovery outcome $\mathrm{e}^{23}$. Improvement in coherence in the beta frequency band were reported to have linear relation with motor function improvement ${ }^{24}$. However, the frequency of alpha rhythm oscillations is associated with motor execution ${ }^{25}$ and beta frequency is modulated during motor execution ${ }^{26}$. These waveforms are suitable to assess neural activities during motor execution state but not the cognitive neural process of motor anticipation which takes place prior to the motor execution. Contingent negative variation (CNV) is a slow negative event-related potential (ERP) that can reflect the cognitive process of event-related motor preparation ${ }^{27-29}$. It is considered as a reliable index for observing cortical excitability of motor anticipatory 22 and can be used to assess the cortical excitability during motor anticipation in stroke patients ${ }^{30}$. Study on source analysis indicated CNV is generated from multiple sources, including cortical and subcortical generators of the anterior cingulate cortex, supplementary motor are and primary motor area $27,31,32$. Stroke survivors with chronic hemiparesis demonstrated significantly enhanced latency and increased amplitude of late CNV at midline during paretic and non-paretic hand preparation ${ }^{27}$ which indicated greater anticipatory effort in response to task execution. This is given further support by our previous study which observed early CNV onset time with increased peak amplitudes of bilateral hemispheres in people with subacute stroke ${ }^{31}$. The 
study observed similar amount of computational demand from the contraleisional and ipsileisional hemisphere during motor planning phase, as reflected in the lack of significant difference in CNV latency and peak amplitude.

Virtual reality (VR) plays a prominent role in promoting functional recovery post stroke. It provides goal-orientated tasks and enriched motivational training to improve patients' ability to implement the plan ${ }^{32-34}$. In addition, it has the potential to implement effective intervention at low cost ${ }^{35}$. The impact of VR intervention on motor planning process was reported in study that utilized VR combined robotic gait training ${ }^{36}$. The study observed strong mu rhythm and event-related-spectral-perturbations post VR intervention which were associated with clinical improvement in gait. This study offers some insight on the potential benefit of VR intervention on motor planning and subsequent functional recovery. To date, there is limited evidence on whether cognitive neural process of motor planning may be improved by VR intervention in and whether changes in cognitive neural process may contribute to upper limb functional improvement. The aims of the present study were to investigate the impact of VR intervention on cortical excitability, motor anticipation and upper limb function in people with subactue stroke. This study hypothesised that patients who underwent VR training had significant reduction in CNV latency and mean peak amplitude when executing palmar grasp movement with the paretic hand. Upper limb function would also be improved significantly post intervention. Patients who received VR intervention have significantly more improvement that in patients who conventional intervention.

\section{Methods}

\subsection{Study setting}

This trial was a parallel randomized, single-blinded, controlled trial. Data collection took place in the electrically shielded Brain Laboratory of the First Affiliated Hospital, Sun Yat-sen University, Guangzhou, China. No change to the study design was made after commencement.

\subsection{Ethics}

This study was approved by the Ethical Committee of The First Affiliated Hospital of Sun Yat-sen University. All participants were provided with a comprehensive explanation of the experimental procedure and a participant information sheet. Participants were asked to provide written consent prior to study enrolment. The study was on the Chinese Clinical Trial Registry (http://www.chictr.org.cn/searchprojen.aspx; Registration No.: ChiCTR-IOC-15006064; Retrospective registration: May 2015)

\subsection{Recruitment and sample size}

Recruitment took place between July 2016 and June 2018 in the inpatient ward of the Department of Rehabilitation Medicine, The First Affiliated Hospital of Sun Yat-sen University. The inclusion criteria were: (1) first ever occurrence of stroke within 1 to 6 months; (2) stroke occurrence confirmed by magnetic resonance imaging (MRI) or computed tomography (CT); (3) age between 40 and 80 years old; (4) have at least $20^{\circ}$ of wrist flexion/extension and at least $10^{\circ}$ of finger flexion and extension of the paretic limb; (5) be able to sit at least 30 minutes without assistance; and (6) no severe cognitive impairment (Mini Mental State Examination $>21)^{37}$. The exclusion criteria were: (1) large area of cerebral infarction; (2) brainstem injury; (3) hand deformity; (4) visual field deficits; and (5) have already received elements of the intervention program over 1 week. Suitable participants were identified by the clinical team during the routine medical admission procedure. Patients were then approached by a member of the research team who was not involved in providing medical care to explain about the study. All approached patients were asked to contact a member of the research team to express their interest.

Sample size was based on a pilot trial where 16 subacute stroke participants were allocated into VR and control group. CNV latency during paretic hand movement post intervention was used as primary outcome measure for sample size calculation. The calculation of sample size was conducted in the software GPower ver 3.1.2, using the "A priori: Compute required sample size -given a, power, and effect size" as type of power analysis. Preliminary results indicated a mean CNV latency of $1740.22 \mathrm{~ms}$ (SD 120.78) for the control group and $1614 \mathrm{~ms}$ (SD 122.61) for VR group post intervention $₫$ which gave an effect size of 0.50 . With a error probability of 0.05 and a power of $0.95 \llbracket a$ sample size of 36 was sufficient for the present study. Figure 1 shows the number of participants at each stage of the study.

\subsection{Randomization, Concealment, and Blinding}

Participants were randomly allocated to either VR group or control group in a 1:1 ratio by simple randomization. The randomization schedule was calculated in statistical software SPSS (IBM SPSS statistics version 20, USA) by a statistical expert from the Faculty of Medical Statistics and Epidemiology, Sun Yat-sen University. The sequence of allocation were kept in sealed envelopes and revealed by a member of the research team after the participant was enrolled. Participants were blinded from their group allocation but were informed that they had an equal chance of allocation to the VR or control group before study participation. The outcome assessor was also blinded to 
group allocation. The treating therapists were not blinded to group allocation. There was no planned interim analyses or stopping rules as power calculation was conducted to enable analysis

\subsection{Procedure}

Interventions were delivered for 2 weeks with 5 training sessions per week. Each session lasted for 60 minutes. Designated therapists provided either VR training or occupational therapy for upper limb function in addition to routine medical care and other rehabilitation deemed necessary by treating physicians.

\subsection{Outcome measures}

Primary outcome measure was CNV latency. Secondary outcome measures were EMG onset time, CNV peak amplitude and functional scales. CNV peak amplitude was adopted to assess the cortical excitability during the motor planning phase. Clinical assessment scales of Action Research Arm Test (ARAT) ${ }^{38}$ (range: 0-57); Upper Limb Fugl-Meyer Assessment (FMA-UL) ${ }^{39}$ (range: 0-66); and National Institutes of Health Stroke Scale (NIHSS) (range: 0-40) were adopted to assess functions. Outcome measures were not altered after commencement of the study. All outcome measures were assessed before and after intervention.

\subsection{Intervention}

The VR intervention was delivered through an interactive training system (A2, YiKang Ltd., China) which comprised of a weight support exoskeleton arm with high precision displacement velocity sensors. Graded upper limb VR training exercises were provided to train the motion of reaching and reach to grasp. Parameters such as grasp power, object moving speed, interval between objects, lateral left or right dispersion of object start positions were adjustable to suit participants functional capability and progressive difficulty level. During VR intervention, participants were seated in front of a table facing the monitor, with the arms in the weight support exoskeleton arm. Figure $2 \mathrm{~A}$ illustrates the setting of the VR system.

Participants in the control group received conventional occupational therapy that consisted of task-related practice of gross movements and dexterity, including grip and selective finger movement, and activities of daily living. Training frequency was matched to those in the VR group.

\subsection{ERP paradigm}

The ERP experimental procedure and paradigm adopted in the present study was in accordance with a published study ${ }^{31}$. Participants were seated in front of a table in an electrically shielded laboratory with his/her shoulder flexed to 0 and $10^{\circ}$, elbows flexed to $130^{\circ}$ and wrists orientated in a neutral position. The motion of palm opening and closing of the hand occurred in the horizontal plane. Figure $2 \mathrm{~B}$ illustrates the staring position of the experiment. All of the participants were first fully explained on the procedure of the experiment. This was followed by 5 to 10 minutes of practice to familiarise with the protocol. Signal recording then began.

The EPR paradigm was as follow: A white fixation point "+" first appeared in the centre of the screen for 500 ms. Then, visual and auditory cues (S1) were given simultaneously for $2000 \mathrm{~ms}$. A picture cueing grasp motion with either the left or right hand was displayed on the screen, accompanied by an auditory cue to indicate right or left side palmar grasp. During $S 1$, the participants were required to judge the palmar grasp task. Then a grey reaction window (S2) of 3000 ms appeared, the participants performed left or right palmar grasp, and avoid making compensatory movements. Then a dark screen of 2000 ms appeared, the participants restored their fingers, and then entered the next trial. The experiment consisted of 40 trials for each hand, totalling 80 trials. The order of the trials were randomised. A diagram of the ERP paradigm can be found in previous published study ${ }^{31}$.

\subsection{EEG}

EEG activities were recorded by a 32-channels QuickAmp amplifier and Ag/AgCl scalp electrodes (BrainProducts, Germany). The electrodes were positioned in accordance with the international 10-20 system. Electrodes were filled with conductive gel to maintain the impedance below $5 \mathrm{k} \Omega$ EEG. EMG signals were recorded in DC mode and sampled at $1000 \mathrm{~Hz}$ synchronize with event markers.

Regional of interest

Six electrodes of interest related to motor function were extracted for EEG analysis (left hemisphere: F3 and C3, right hemisphere: F4 and C4, midline region: Fz and Cz). The F3, F4 and Fz lies over the pre-motor cortex and the C3, C4 and Cz lies over the primary motor cortex. Figure $2 \mathrm{C}$ illustrates the locations of the electrodes. For the purpose of statistical comparison, the left and right side hemispheres were flipped right to left in participant with right hemispheric lesion so that the "left" hemisphere was always the lesioned hemisphere ${ }^{22}$.

Page $4 / 15$ 


\subsection{EMG}

The EMG activities were simultaneously recorded with EEG. Two surface electrodes were placed along the extensor digitorum using 2 surface electrodes with a 2-cm inter-electrode distance. The EMG reaction time was measured with the time from S1 to the EMG onset as shown in Figure 3A.

\subsection{Signal processing}

EEG signals were new referenced to the bilateral mastoid. Eye movement artefacts were removed through the Ocular Correction Independent Component Analysis (ICA) as part of standard operating procedure ${ }^{14}$. EEG and EMG signals were filtered using a $50 \mathrm{~Hz}$ notch filter and a bandpass filter from 0.1 to $30 \mathrm{~Hz}$. In order to acquire stimulus-locked ERPs, the EEG and EMG signals were segmented into epochs of 500 ms pre to 3000 ms post aligned to S1. The baseline was corrected according to the first 200 ms of the epochs, which was the 200 ms time window before $\mathrm{S} 1$ onset.

The EMG reaction time was measured with the time from S1 to the EMG onset. As the maximum CNV amplitude was detected between 1300 ms to 1800 ms after S1, peak detection was used to detect the maximum CNV amplitude in this time window. The maximum amplitude must be higher than the amplitude of 2-3 points on the front and back sides ${ }^{40}$. The CNV latency was calculated as the time from $\mathrm{S} 1$ to the maximum amplitude. The mean CNV amplitude was calculated from 1300 to 1800 ms to acquire the average amplitude of the CNV potential (Figure 3B).

\subsection{Statistical analysis}

Statistical analyses were performed using SPSS version 22 (IBM SPSS statistics version 20, USA). The Wilcoxon signed-rank test was applied to verify the statistical significance of the changes in the ARAT, FMA-UL, and NIHSS within groups, and Mann-Whitney U test was conducted to compare between the two groups. For EMG reaction time and CNV latency analyses, three-way mixed analysis of variance (ANOVA) were calculated, with task (paretic hand movement vs. non-paretic hand movement) and TIME (baseline vs. post-training) as within-subject factors, GROUP (VR group vs. control group) as between-subject factor. Four-way mixed ANOVA, with TASK (paretic hand movement vs. non-paretic hand movement), ELECTRODE (F3, C3, F4, C4, Fz, and Cz) and TIME (baseline vs. post-training) as within-subject factor, TREATMENT (VR group vs. control group) as between-subject factor were performed to assess the training effects. The GreenhouseGeisser adjustment was applied to adjust the degrees of freedom if the assumption of the Mauchly's test of sphericity was not significant. Separate mixed model ANOVAs were tested for each level of electrodes. If there was a significant interaction in the within-subject factor and between-subject factor, then subsequent independent sample $t$-tests were performed to further investigate the differences between the two groups. The significance level for all statistical analysis was set at 0.05 . Bonferroni-adjusted significance tests were performed to correct the $p$-values of electrodes for multiple comparisons. Thus, the corrected significance level for electrode was $a=0.05 \div 6=0.008^{41}$.

\section{Results}

\subsection{Demographics}

Recruitment for this study took place between January 2017 till December 2019. A total of 36 participants with upper limb hemiplegia resulting from a unilateral subcortical lesion of the first occurrence of stroke were recruited. No harmful event or unintended effect was reported in each group. All participants completed the study and the number of participants included in the analysis was as planned. All recruited participants were included in the data analysis. Eighteen participants were allocated to the VR group and 18 participants were allocated to the control group. Table 1 gives a summary of the demographic data and clinical characteristics of the sample cohorts. Two independent sample $t$-test showed no significant difference in the number of cases, sex and age between the VR group and the control group $(p>0.05)$. Mann-Whitney U tests showed no significant difference in the FMA-UL, ARAT, and NIHSS scores between two groups before treatment $(p>0.05)$. Table 2 provides a summary of the results of the clinical scales pre and post intervention for both groups.

Table 1. Demographics and clinical characteristics of the sample cohorts. 


\section{VR group ( $\mathrm{n}=18)$ control group $(\mathrm{n}=18)$}

\begin{tabular}{ccc}
\hline $\begin{array}{l}\text { Age (SD), } y \\
\text { Sexø } \boldsymbol{n}(\%)\end{array}$ & $57.8(8.4)$ & $58.4(9.3)$ \\
\hline Male & $10(55.6)$ & $10(55.6)$ \\
\hline Female & $8(44.4)$ & $8(44.4)$ \\
\hline FMA-UL & $18.55(5.97)$ & $18.33(5.17)$ \\
\hline ARAT & $26.33(6.04)$ & $26.00(7.09)$ \\
\hline NIHSS & $5.44(2.91)$ & $5.39(2.85)$ \\
\hline MMSE & $26.05(2.64)$ & $25.61(2.87)$ \\
\hline
\end{tabular}

\subsection{Behavioural data}

\subsubsection{EMG reaction time}

The mixed model ANOVA analysis indicated a significant main effect of TIME on the reaction time $[F(1,34)=483.33, p=0.00]$, TIMExTREATMENT interaction $[\mathrm{F}(1,34)=5.680, p=0.00]$ and TIMExTASK interaction $[\mathrm{F}(1,34)=14.943, p=0.00]$. Thus, separate ANOVAs were calculated for each level of TIME and TREATMENT. Further analysis showed a statistically significant main effect of Task at baseline $[\mathrm{F}(1,34)=21.10, p=0.00]$ reaction time of the non-paretic hand was earlier than the paretic hand in both groups $(p=0.00)$. A significant main effect of Task was observed after treatment $[F(1,34)=5.29, p=0.03]$. The reaction time of non-paretic hand and paretic hand in both groups was earlier after treatment. In the VR group, paretic hand was $t=15.77 \rrbracket p=0.00$, non-paretic hand was $t=10.27 \rrbracket p=0.00$. In the control group, paretic hand was $t=23.05 \rrbracket p=0.00$, non-paretic hand was $t=6.08 \rrbracket p=0.00$. The reaction time of paretic hand in VR group was earlier than those observed in control group after training $(\mathrm{t}=2.18 \otimes p=0.04)$. No significant difference in the reaction time of non-paretic hand between two groups after training $(t=1.55 \rrbracket p=0.13)$. Table 2 presents the comparison of EMG reaction time before and after intervention for both groups.

\subsubsection{CNV latency}

There was a significant TIMEXTREATMENT interaction $[\mathrm{F}(1,34)=4.86, p=0.03]$, and main effect of $\mathrm{TIME}[\mathrm{F}(1,34)=339.54 \rrbracket p=0.00]$. However, there was no significant TASK $\times$ TIMExTREATMENT interaction $[F(1,34)=0.447 \rrbracket p=0.508]$. There was no significant difference in CNV latency of the non-paretic hand or paretic hand movement between two groups at baseline $(\mathrm{TASK}[\mathrm{F}(1,34)=2.01 \rrbracket p=0.17]$, and at TASK $\times$ TREATMENT interaction $[F(1 \otimes 34)=0.01 \rrbracket p=0.91])$. Analysis indicated a significant main effect of TASK [F(1区34) $=5.286 \otimes p=0.03]$. There was no significant TASK $\times$ TREATMENT interaction $[\mathrm{F}(1 \otimes 34=0.44 \rrbracket p=0.51]$, and main effect of TASK [F(1,34) $=0.00 \rrbracket p=1.00]$. Then, subsequent paired sample t-tests revealed CNV latency of non-paretic hand and paretic hand in both groups was earlier after treatment (VR group: paretic hand: $\mathrm{t}=8.04 \rrbracket p=0.00$, non-paretic hand: $\mathrm{t}=11.78 \otimes p=0.00$; control group: paretic hand: $\mathrm{t}=11.89 \otimes p=0.00$, non-paretic hand: $\mathrm{t}=11.11 \otimes p=0.00)$. The CNV latency of paretic hand in the VR group showed earlier than control group $(\mathrm{t}=2.41 \otimes p=0.02)$. No significant difference in the CNV latency of non-paretic hand between the two groups was observed $(\mathrm{t}=1.15 \rrbracket p=0.26)$. Table 3 presents the comparison of CNV latency before and after intervention for both groups during non-paretic and paretic hand movements.

\subsection{CNV amplitude}

The mixed model ANOVA analysis on the mean CNV amplitude between the VR group and control group during non-paretic hand and paretic hand movement revealed a significant TASK $\times$ TIMExTREATMENT×ELECTRODE interaction $[\mathrm{F}(5,17)=6.91 \rrbracket p=0.00]$, TASK $\times$ TIME interaction $[F(1,34)=156.17, p=0.00]$, and TASK $\times E L E C T R O D E$ interaction $[F(5,17)=4.383, p=0.00]$. The mean CNV amplitude was modulated by TIME condition $[\mathrm{F}(1,34)=501.29 \otimes p=0.00]$ and ELECTRODE condition $[\mathrm{F}(5,17)=6.063, p=0.00]$. Therefore, TIME, TRANTMENT and TASK were tested for individual effects. Before training, There was no significant difference in mean CNV amplitude of non-paretic hand and paretic hand between VR group and control group were observed $(T A S K \times T R E A T M E N T \times E L E C T R O D E$ interaction $[F(1,34)=0.27 \rrbracket p=0.61])$. Further investigation revealed that there was a significantly larger mean CNV amplitude at midline (Fz, Cz) and ipsilateral (C4) regions when conducting paretic hand movement in both groups (p叉0.05). There was a significantly larger mean CNV amplitude at midline (Fz, $\mathrm{Cz}$ ) and contralateral (C4) regions when conducting non-paretic hand movement in both groups. The reduction in mean CNV amplitude between VR group and control group after treatment was analyzed by mixed ANOVA. There was a significant TASK×TREATMENT×ELECTRODE interaction $[F(5,17)=3.560 \unrhd p=0.03]$ after treatment. Further investigation of these effects revealed that the mean CNV amplitude declined during non-paretic hand movement and paretic hand movement in both groups after intervention [VR group: paretic hand: $F(1,34)=2.744 \rrbracket p$ 
$=0.033$, non-paretic hand: $\mathrm{F}(1,34)=5.34 \rrbracket p=0.00$; control group: paretic hand: $\mathrm{F}(1,34)=7.80 \rrbracket p=0.00$, non-paretic hand: $\mathrm{F}(1,34)=7.94 \rrbracket p$ $=0.00$, with significant difference in decrease in midline $(\mathrm{Fz}$ and $\mathrm{Cz})$ regions during paretic hand movement between VR group and control group ( $p \otimes 0.05$ ), and in the decrease in midline ( $\mathrm{Fz}$ and $\mathrm{Cz}$ ) 『ipsilateral (C3) and contralateral (C4) regions during non-paretic hand movement between VR group and control group ( $p \otimes 0.05$ ). Table 4 presents the changes in CNV mean amplitude in VR and control group during nonparetic hand and paretic hand movements. Figure 4 shows the topographic maps of participants in two groups when executing paretic and non-paretic hand movement tasks. Figure 5 and Figure 6 illustrate the CNV amplitudes of the VR group during paretic and non-paretic hand movements. CNV amplitudes during paretic and non-paretic hand movements are illustrated in Figure 7 and Figure 8.

\subsection{Clinical functions}

The scores of FMA-UL, ARAT and NIHSS post-treatment decreased significantly in both groups after intervention $(p<0.05)$. The improvements in ARAT and FMA-UL in the VR group were significantly higher than the control group [FMA-UL: $Z=-2.34, p=0.02 ; A R A T: Z=$ $-2.09, p=0.04]$. No significant difference in NIHSS scores was observed between the VR and control groups $[Z=-1.80, p=0.07]$. Table 5 presents a summary of the changes in NIHSS, FMA-UL and ARAT in the VR group and control group.

Table 2: Comparison of EMG reaction time before and after intervention during non-paretic and paretic hand movements.

\begin{tabular}{|c|c|c|c|c|c|c|c|c|c|c|}
\hline \multicolumn{6}{|c|}{ Paretic hand } & \multicolumn{5}{|c|}{ Non-paretic hand } \\
\hline & Pre $(\mathrm{ms})$ & Post (ms) & $t$ & $p$ & $95 \% \mathrm{CI}$ & Pre (ms) & Post (ms) & $t$ & $p$ & $\begin{array}{c}95 \% \\
\text { CI }\end{array}$ \\
\hline & $\begin{array}{c}2526.3 \\
\pm 121.78\end{array}$ & $\begin{array}{c}2472.06 \pm \\
117.28\end{array}$ & 10.27 & 0.00 & $\begin{array}{l}-26.74- \\
135.24\end{array}$ & $1661.33 \pm 134.48$ & $\begin{array}{c}1533.00 \pm \\
117.88\end{array}$ & 10.27 & 0.00 & $\begin{array}{l}-24.90- \\
106.46\end{array}$ \\
\hline ll & $2532.19 \pm 122.4$ & $2486.56 \pm 121.75$ & 23.05 & 0.00 & $\begin{array}{l}37.07- \\
128.32\end{array}$ & $1654.56 \pm 197.5$ & $\begin{array}{l}1565.94 \\
\pm 197.77\end{array}$ & 6.08 & 0.00 & $\begin{array}{l}50.83- \\
112.46\end{array}$ \\
\hline
\end{tabular}

Table 3: Comparison of CNV latency before and after intervention during non-paretic and paretic hand movements.

\begin{tabular}{|c|c|c|c|c|c|c|c|c|c|c|}
\hline$p$ & & Paretic hand & & & & & Jon-paretic hand & & & \\
\hline & Pre (ms) & Post (ms) & $t$ & $p$ & $\begin{array}{c}95 \% \\
\text { CI }\end{array}$ & Pre (ms) & Post (ms) & $t$ & $p$ & $\begin{array}{c}95 \% \\
\text { CI }\end{array}$ \\
\hline & $1712.33 \pm 143.99$ & $\begin{array}{l}1593.39 \\
\pm 134.39\end{array}$ & 15.77 & 0.00 & $\begin{array}{l}12.63- \\
211.15\end{array}$ & $1661.33 \pm 134.48$ & $1553.00 \pm 117.88$ & 10.29 & 0.00 & $\begin{array}{l}18.23- \\
193.88\end{array}$ \\
\hline rol & $1714.33 \pm 191.95$ & $1631.56 \pm 193.41$ & 23.05 & 0.00 & $\begin{array}{l}-51.53- \\
217.09\end{array}$ & $1654.56 \pm 197.77$ & $1565.94 \pm 197.77$ & 6.084 & 0.00 & $\begin{array}{l}-49.15- \\
226.37\end{array}$ \\
\hline
\end{tabular}

Table 4: Changes in CNV mean amplitude in VR and control group during non-paretic hand and paretic hand movements. 


\begin{tabular}{ccccc|cccc}
\hline \multicolumn{5}{c|}{ Non-paretic hand movement $(\mathrm{mV})$} & \multicolumn{4}{c}{ Paretic hand movement $(\mathrm{mV})$} \\
\hline Electrodes & VR group & Control group & $\boldsymbol{p}$ & $95 \%$ CI & VR group & Control group & $\boldsymbol{p}$ & $95 \%$ CI \\
\hline C3 & $0.88 \pm 0.55$ & $0.42 \pm 0.21$ & 0.00 & $0.29-0.79$ & $0.75 \pm 0.53$ & $0.56 \pm 0.26$ & 0.00 & $-0.14-0.44$ \\
Cz & $0.96 \pm 0.41$ & $0.64 \pm 0.23$ & 0.00 & $0.14-0.59$ & $1.10 \pm 1.18$ & $0.64 \pm 0.24$ & 0.00 & $-0.19-1.03$ \\
C4 & $0.85 \pm 0.34$ & $0.53 \pm 0.26$ & 0.00 & $0.11-0.53$ & $0.77 \pm 0.59$ & $0.48 \pm 0.22$ & 0.00 & $0.46-0.63$ \\
F3 & $0.72 \pm 1.25$ & $0.45 \pm 0.29$ & 0.35 & $-0.51-0.72$ & $0.81 \pm 0.52$ & $0.61 \pm 0.28$ & 0.35 & $-0.10-0.48$ \\
Fz & $1.18 \pm 0.87$ & $0.53 \pm 0.29$ & 0.00 & $0.22-1.10$ & $0.96 \pm 0.53$ & $0.75 \pm 0.29$ & 0.00 & $-0.38-0.54$ \\
F4 & $0.93 \pm 0.92$ & $0.44 \pm 0.29$ & 0.03 & $0.01-0.94$ & $0.87 \pm 0.72$ & $0.41 \pm 0.29$ & 0.03 & $0.15-0.89$ \\
\hline
\end{tabular}

Table 5: A summary of the changes in NIHSS, FMA-UL and ARAT in the VR group and control group.

\begin{tabular}{cccc}
\hline group & NIHSS & FMA-UL & ARAT \\
\hline VR group & $2.167 \pm 0.83$ & $4.72 \pm 0.87$ & $5.06 \pm 0.91$ \\
Control group & $1.83 \pm 0.50$ & $3.89 \pm 1.15$ & $4.11 \pm 1.20$ \\
$\mathbf{Z}$ & -1.80 & -2.338 & -2.09 \\
$\boldsymbol{p}$ & 0.07 & 0.02 & 0.04 \\
$\mathbf{9 5 \%}$ CI & $-0.15-0.82$ & $0.12-1.44$ & $0.20-1.69$ \\
\hline
\end{tabular}

\section{Discussion}

The present study investigated the impact of VR intervention on motor anticipation in people with subacute stroke and explored the relationship between changes of cortical excitability and upper limb function. The main findings included that EMG response time was significantly shorter in the VR group than the control group during paretic hand movement post intervention. The reduction of CNV latency and peak amplitude in the VR group was significantly lower than the control group. The improvements of upper limb motor function were significantly higher in the VR group than the control group after treatment.

\subsection{The Effect of VR training on Behavioural Data}

\section{EMG onset and CNV latency}

Reaction time is attributed to the effect of muscle onset and CNV latency is related to the brain's computational demand and cognitive processing speed when executing a movement ${ }^{42-44}$. Impairments in motor anticipation among subacute stroke patients could be manifested as a delay in EMG reaction time and increased preparation time of cerebral hemispheres. This is supported by neural electrophysiological study which indicated a significant increase in cortical preparatory activation correlated with a behavioral enhancement ${ }^{45}$. In this study, the EMG response time and CNV latency decreased after intervention in both groups, which indicated that EMG onset was earlier and the motor anticipation time of bilateral cerebral hemispheres was shortened in both groups. Previous studies reported that subacute stroke patients demonstrated typical response-priming effect, with extended reaction time and CNV latency ${ }^{22,31}$. The shorter reaction time might be related to improvement in the neurocognitive process to plan an upcoming task. The larger reduction in reaction time in the VR group than those observed in the control group during paretic hand movement post training suggested that VR intervention may be more effective than conventional therapy training to improve cognitive neural process. Task oriented VR training requires the user to have an understanding of the upcoming task to be able to complete the task which in theory is able to improve cognitive function. The reduction in EMG reaction time and CNV latency shortened and tended to approach normal level post intervention, indicating an improvement in neural activation efficiency of motor anticipation and the improvement of motor dynamics of upper limb function ${ }^{46}$. Therefore, VR training may improve hemiplegic upper limb motor anticipation by promoting neuronal activation in stroke patients.

\section{Upper Limb Function}

Both the ARAT and FMA-UL demonstrated significantly higher improvements in upper limb motor function after VR training compared with control group after treatment. These findings indicate that VR training is superior to the conventional occupational training in promoting motor function of the upper limb. The improvement in upper limb function induced by VR intervention is consistent with the results 
published from systematic review ${ }^{8}$ with indicated a small to medium effect in favor of VR intervention when compared with control therapy. A published study indicated ARAT score was correlated with smaller CNV peak amplitude among the subacute stroke population.

\section{CNV amplitude}

CNV amplitude is an index of anticipation and reflects the amount of cognitive neural resource ${ }^{47}$ required to plan an upcoming task ${ }^{29,48}$. Early literature reported cerebral activity of the contralesional hemisphere in the early stage of stroke during movement of the paretic side $^{49,50}$. A published study reported that subacute stroke patients recruit additional neural resources from the contralesional hemisphere during the motor planning phase of grasp motion 27,31 , as indicated in the increase in CNV amplitude in the lesional and contraleisonal hemispheres during paretic hand movement. Previous study reported expansion of the cortical mapping in animal ${ }^{51}$ and human ${ }^{52}$ after cortical damage. The expansion of cortical mapping during early stage of stroke is due to the neuroplasticity process related to the reacquisition of motor skills $s^{53}$ and map expansion was reported to continue up to 8 weeks after training had stopped ${ }^{54}$. Once the sequence of the motor task is learned, the mapping representation tends to return to its original size (REF). Finding of the present study suggested that VR intervention was effective in reducing the cognitive resources from the contralesional hemisphere during paretic hand movement. The reduction in CNV amplitude post intervention is also consistent with MRI studies suggested that neuroplasticity recovery manifests as a decrease in sensorimotor cortex activation on the contralesional hemisphere ${ }^{49}$, which can be interpreted as an increase in the efficiency of neural activation. Correlation between the recovery of motor function and re-lateralization of activation in the contralateral hemisphere was also reported ${ }^{55}$. Thus, the reorganization of motor control occurs after stroke and may involve the ipsilateral or contralateral cortex, depending on the location and size of the brain lesion and theoretically on the somatotopic organization of the residual pyramidal tracts ${ }^{56}$. The observed significantly larger reduction of CNV amplitude in the VR group during paretic movement provides further support that VR intervention may be effective in reducing hyperactivity of the contralesional hemisphere.

Our previous EEG study indicated that significant increase in cortical activity at contralesional C4 and ipsilesional C3 electrodes during nonparetic hand movement ${ }^{31}$. The increased in bilateral cortical activity is related to the compensatory over activation of the contraleisional hemisphere and the lack of cross hemispheric inhibition from the lesional to non-lesional hemisphere. The present study observed significantly larger reduction in CNV amplitude in midline『contraleisonal C4 and ipsilateral C3 Fz and Cz electrodes during non-paretic hand movement in the VR group than control group. The reduction in CNV amplitude corresponds with improvement in upper limb function which provides further support that VR intervention may be effective in reducing interhemispheric misbalance than conventional training.

\section{Limitations}

The data in the present study should be interpreted with cautious due to its limitations. The study included participants at the subacute stage of stroke which limits the external validity of the finding. Future research should include participants at the acute and chronic stage of stroke to enhance the external validity of the findings. The right to left flipping of the scalp site in participants with right hemisphere lesion site may be considered as a limitation. However, it is not uncommon in published literature that involve either EEG or fMRI study to perform the flipping as a mean to increase statistical power. The present study did not involve a follow up period and it remains unclear if the observed benefit may carry on in longer term.

\section{Conclusions}

This study investigated the electrophysiological changes of motor anticipation of palmar grasp motion induced by VR intervention in people with subacute stroke. The larger reduction in reaction time, CNV latency and mean CNV peak amplitude in the VR intervention group are indicative of more reduction in brain computational demand during palmar grasp task. Clinical function in the VR group was also larger in the VR group which suggest that VR intervention may be more beneficial in improving upper limb function patients with subacute stroke. Improvement in upper limb function may be related to improvement of the neural anticipatory process.

\section{Declarations}

\section{Ethics}

This study was approved by the Ethical Committee of The First Affiliated Hospital of Sun Yat-sen University (Ethics no.: [2014] 88). All participants were provided with a comprehensive explanation of the experimental procedure and a participant information sheet. Participants were asked to provide written consent prior to study enrolment. Tria/ registration: Chinese Clinical Trial Registry (Registration No.: ChiCTR-IOC-15006064). Registered on 11 May 2015. http://www.chictr.org.cn/searchprojen.aspx 
Not applicable

\section{Availability of data and materials}

The data that support the findings of this study are available from The First Affiliated Hospital, Sun Yat-sen University but restrictions apply to the availability of these data, which were used under license for the current study, and so are not publicly available. Data are however available from the authors upon reasonable request and with permission of The First Affiliated Hospital, Sun Yat-sen University.

\section{Competing interests}

The authors declare that they have no competing interests.

\section{Funding}

This study was supported by the National Natural Science Foundation of China (Grant No.: 81971224), China Postdoctoral Science Foundation Funded Project (Grant No.: 2019M652867) 『Scientific Research Project of Guangdong Traditional Chinese Medicine Bureau (Grant No.: 20201118). The publication of the manuscript was supported by the Health Commission of Guangdong Province (Grant No.:2018KT1673) and Sun Yat-sen University Clinical Research 5010 funding programme (Grant No. 2014001). All funding bodies did not influence the design of the study and collection, analysis and interpretation of the data and in writing the manuscript.

\section{Authors' contributions}

All authors have read and approved the final manuscript. All authors meet the four primary ICMJE criteria for authorship. In addition, all authors have been actively involved in the study in different capacities: LC and WLAL designed the study and conducted all stages of the study including data collection, analysis, interpretation, and drafting of the manuscript. YC participated in the design of the research protocol, data analysis and drafting of the manuscript. WLAL and LC revised the manuscript, interpreted the data and managed the trial.

\section{Acknowledgements}

We would like to express our gratitude to everyone who participated in the described study.

\section{Authors' information}

Dr. Ling Chen

E-mail『chenling@gzucm.edu.cn

Department of Acupuncture and Moxibustion, The Second Affiliated hospital of Guangzhou University of Chinese Medicine, Guangzhou, China

Second Clinical College, Guangzhou University of Chinese Medicine, Guangzhou, China

Dr. Yi Chen

Email: yytyzl@gzucm.edu.cn

Guangzhou University of Chinese Medicine, Guangzhou, China

Dr. Wai Leung Ambrose Lo

Department of Rehabilitation囚The First Affiliated Hospital, Sun Yat-sen University

\section{References}

1. Vorkas PA, Shalhoub J, Lewis MR, et al. Metabolic Phenotypes of Carotid Atherosclerotic Plaques Relate to Stroke Risk: An Exploratory Study. Eur J Vasc Endovasc Surg. 2016;52(1):5-10.

2. Global, regional, and national disability-adjusted life-years (DALYs) for 315 diseases and injuries and healthy life expectancy (HALE), 1990-2015: a systematic analysis for the Global Burden of Disease Study 2015. Lancet. 2016;388(10053):1603-1658.

Page 10/15 
3. Jorgensen HS, Nakayama H, Raaschou HO, Olsen TS. Stroke. Neurologic and functional recovery the Copenhagen Stroke Study. Physical medicine and rehabilitation clinics of North America. 1999;10(4):887-906.

4. Feigin VL, Lawes CM, Bennett DA, Anderson CS. Stroke epidemiology: a review of population-based studies of incidence, prevalence, and case-fatality in the late 20th century. The Lancet. Neurology. 2003;2(1):43-53.

5. Hakkennes S, Keating JL. Constraint-induced movement therapy following stroke: a systematic review of randomised controlled trials. Aust J Physiother. 2005;51(4):221-231.

6. Kwakkel G, Kollen BJ, van der Grond J, Prevo AJ. Probability of regaining dexterity in the flaccid upper limb: impact of severity of paresis and time since onset in acute stroke. Stroke. 2003;34(9):2181-2186.

7. Rosamond W, Flegal K, Friday G, et al. Heart disease and stroke statistics--2007 update: a report from the American Heart Association Statistics Committee and Stroke Statistics Subcommittee. Circulation. 2007;115(5):e69-171.

8. Aminov A, Rogers JM, Middleton S, Caeyenberghs K, Wilson PH. What do randomized controlled trials say about virtual rehabilitation in stroke? A systematic literature review and meta-analysis of upper-limb and cognitive outcomes. Journal of Neuroengineering \& Rehabilitation. 2018;15(1):29.

9. Mullick AA, Subramanian SK, Levin MF. Emerging evidence of the association between cognitive deficits and arm motor recovery after stroke: A meta-analysis. Restorative neurology and neuroscience. 2015;33(3):389-403.

10. Varghese JP, Merino DM, Beyer KB, Mcllroy WE. Cortical control of anticipatory postural adjustments prior to stepping. Neuroscience. 2016;313:99-109.

11. Svoboda K, Li N. Neural mechanisms of movement planning: motor cortex and beyond. Current Opinion in Neurobiology. $2018 ; 49: 33-41$.

12. Tanji J, Evarts EV. Anticipatory activity of motor cortex neurons in relation to direction of an intended movement. Journal of neurophysiology. 1976;39(5):1062-1068.

13. Bai O, Rathi V, Lin P, et al. Prediction of human voluntary movement before it occurs. Clinical Neurophysiology. 2011;122(2):364-372.

14. Kalaska JF. From intention to action: motor cortex and the control of reaching movements. Adv Exp Med Biol. 2009;629:139-178.

15. Schaefer SY, Haaland KY, Sainburg RL. Hemispheric specialization and functional impact of ipsilesional deficits in movement coordination and accuracy. Neuropsychologia. 2009;47(13):2953-2966.

16. Wetter S, Poole JL, Haaland KY. Functional implications of ipsilesional motor deficits after unilateral stroke. Archives of physical medicine and rehabilitation. 2005;86(4):776-781.

17. Vahdat S, Darainy M, Ostry DJ. Structure of plasticity in human sensory and motor networks due to perceptual learning. The Journal of neuroscience : the official journal of the Society for Neuroscience. 2014;34(7):2451-2463.

18. Hermsdorfer J, Hagl E, Nowak DA. Deficits of anticipatory grip force control after damage to peripheral and central sensorimotor systems. Hum Mov Sci. 2004;23(5):643-662.

19. Nowak DA, Hermsd Rfer J, Topka H. Deficits of predictive grip force control during object manipulation in acute stroke. Journal of Neurology. 2003;250(7):850-860.

20. Takahashi CD, Reinkensmeyer DJ. Hemiparetic stroke impairs anticipatory control of arm movement. Experimental Brain Research. 2003;149(2):131-140.

21. Tan C, Tretriluxana J, Pitsch E, Runnarong N, Winstein CJ. Anticipatory planning of functional reach-to-grasp: a pilot study. Neurorehabil Neural Repair. 2012;26(8):957-967.

22. Yilmaz O, Birbaumer N, Ramos-Murguialday A. Movement related slow cortical potentials in severely paralyzed chronic stroke patients. Frontiers in Human Neuroscience. 2015;8.

23. Finnigan S, van Putten MJ. EEG in ischaemic stroke: quantitative EEG can uniquely inform (sub-)acute prognoses and clinical management. Clin Neurophysiol. 2013;124(1):10-19.

24. Nicolo P, Rizk S, Magnin C, Pietro MD, Schnider A, Guggisberg AG. Coherent neural oscillations predict future motor and language improvement after stroke. Brain : a journal of neurology. 2015;138(Pt 10):3048-3060.

25. Sabate M, Llanos C, Enriquez E, Gonzalez B, Rodriguez M. Fast modulation of alpha activity during visual processing and motor control. Neuroscience. 2011;189:236-249.

26. Willemse RB, de Munck JC, Verbunt JP, et al. Topographical organization of mu and Beta band activity associated with hand and foot movements in patients with perirolandic lesions. The open neuroimaging journal. 2010;4:93-99.

27. Dean PJ, Seiss E, Sterr A. Motor planning in chronic upper-limb hemiparesis: evidence from movement-related potentials. PLoS One. 2012;7(10):e44558. 
28. do Nascimento OF, Nielsen KD, Voigt M. Movement-related parameters modulate cortical activity during imaginary isometric plantarflexions. Exp Brain Res. 2006;171(1):78-90.

29. Leuthold H, Sommer W, Ulrich R. Preparing for action: Inferences from CNV and LRP. Journal Of Psychophysiology. 2004;18(2-3):77-88.

30. Fattapposta F, D'Agostino VC, My F, et al. Psychophysiological aspects of voluntary skilled movement after stroke: a follow-up study. Archives italiennes de biologie. 2008;146(3-4):147-163.

31. Chen L, Mao Y, Ding M, et al. Assessing the Relationship Between Motor Anticipation and Cortical Excitability in Subacute Stroke Patients With Movement-Related Potentials. Frontiers in neurology. 2018;9:881.

32. Levin MF, Weiss PL, Keshner EA. Emergence of virtual reality as a tool for upper limb rehabilitation: incorporation of motor control and motor learning principles. Physical therapy. 2015;95(3):415-425.

33. Laver KE, Lange B, George S, Deutsch JE, Saposnik G, Crotty M. Virtual reality for stroke rehabilitation. The Cochrane database of systematic reviews. 2017;11:Cd008349.

34. Tinga AM, Visser-Meily JM, van der Smagt MJ, Van der Stigchel S, van Ee R, Nijboer TC. Multisensory Stimulation to Improve Low- and Higher-Level Sensory Deficits after Stroke: A Systematic Review. Neuropsychology review. 2016;26(1):73-91.

35. Llorens R, Noe E, Colomer C, Alcaniz M. Effectiveness, usability, and cost-benefit of a virtual reality-based telerehabilitation program for balance recovery after stroke: a randomized controlled trial. Archives of physical medicine and rehabilitation. 2015;96(3):418-425.e412.

36. Calabrò RS, Naro A, Russo M, et al. The role of virtual reality in improving motor performance as revealed by EEG: a randomized clinical trial. Journal of neuroengineering and rehabilitation. 2017;14(1):53.

37. Folstein MF, Folstein SE, McHugh PR. "Mini-mental state". A practical method for grading the cognitive state of patients for the clinician. J Psychiatr Res. 1975;12(3):189-198.

38. Carroll D. A Quantitative Test Of Upper Extremity Function. J Chronic Dis. 1965;18:479-491.

39. Duncan PW, Propst M, Nelson SG. Reliability of the Fugl-Meyer assessment of sensorimotor recovery following cerebrovascular accident. Physical therapy. 1983;63(10):1606-1610.

40. Luck SJ. An Introduction to the Event-Related Potential Technique. Cambridge: MIT Press 2005.

41. Krishef C. Fundamental Statistics for Human Services and Social Work. Boxton: PWS Pubslihers \% 2017-11-29 18:01:00; 1987.

42. Arito $\mathrm{H}$, Oguri $\mathrm{M}$. Contingent negative variation and reaction time of physically-trained subjects in simple and discriminative tasks. Industrial health. 1990;28(2):97-106.

43. Cirillo J, Finch JB, Anson JG. The impact of physical activity on motor preparation in young adults. Neurosci Lett. 2017;638:196-203.

44. Lang W, Obrig H, Lindinger G, Cheyne D, Deecke L. Supplementary motor area activation while tapping bimanually different rhythms in musicians. Exp Brain Res. 1990;79(3):504-514.

45. Smith AL, Staines WR. Cortical and behavioral adaptations in response to short-term inphase versus antiphase bimanual movement training. Exp Brain Res. 2010;205(4):465-477.

46. Saleh S, Bagce H, Qiu Q, Fluet G, Tunik E. Mechanisms of Neural Reorganization in Chronic Stroke Subjects after Virtual Reality Training. Conference proceedings: ... Annual International Conference of the IEEE Engineering in Medicine and Biology Society. IEEE Engineering in Medicine and Biology Society. Conference. 2011;2011:8118-8121.

47. Jankelowitz SK, Colebatch JG. Movement related potentials in acutely induced weakness and stroke. Exp Brain Res. 2005;161(1):104113.

48. Brunia CH, van Boxtel GJ. Wait and see. Int J Psychophysiol. 2001;43(1):59-75.

49. Butefisch CM, Kleiser R, Korber B, et al. Recruitment of contralesional motor cortex in stroke fpatients with recovery of hand function. Neurology. 2005;64(6):1067-1069.

50. Ward NS, Brown MM, Thompson AJ, Frackowiak RS. Neural correlates of motor recovery after stroke: a longitudinal fMRI study. Brain. 2003;126(Pt 11):2476-2496.

51. Sanes JN, Suner S, Lando JF, Donoghue JP. Rapid reorganization of adult rat motor cortex somatic representation patterns after motor nerve injury. Proc Natl Acad Sci U S A. 1988;85(6):2003-2007.

52. Pascual-Leone A, Grafman J, Hallett M. Modulation of cortical motor output maps during development of implicit and explicit knowledge. Science. 1994;263(5151):1287-1289.

53. Karni A, Meyer G, Rey-Hipolito C, et al. The acquisition of skilled motor performance: fast and slow experience-driven changes in primary motor cortex. Proc Natl Acad Sci U S A. 1998;95(3):861-868.

54. Nudo RJ, Plautz EJ, Frost SB. Role of adaptive plasticity in recovery of function after damage to motor cortex. Muscle Nerve. 2001;24(8):1000-1019.

Page 12/15 
55. Feydy A, Carlier R, Roby-Brami A, et al. Longitudinal study of motor recovery after stroke: recruitment and focusing of brain activation. Stroke. 2002;33(6):1610-1617.

56. Green JB, Bialy Y, Sora E, Ricamato A. High-resolution EEG in poststroke hemiparesis can identify ipsilateral generators during motor tasks. Stroke. 1999;30(12):2659-2665.

\section{Figures}

Figure 1

Flow diagram of the study.

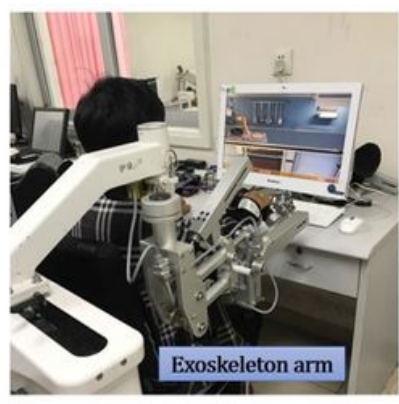

A

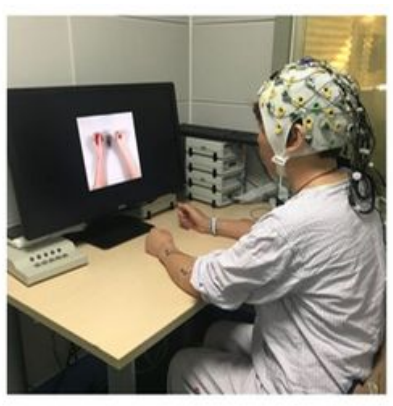

B

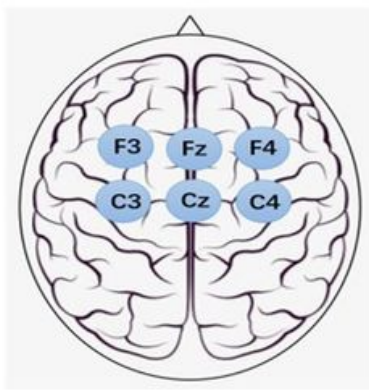

C
A: Exoskeleton arm for VR training system
B: Starting position of EEG and EMG recording
C: locations of the six electrodes

\section{Figure 2}

Diagrams of the experimental set up. 


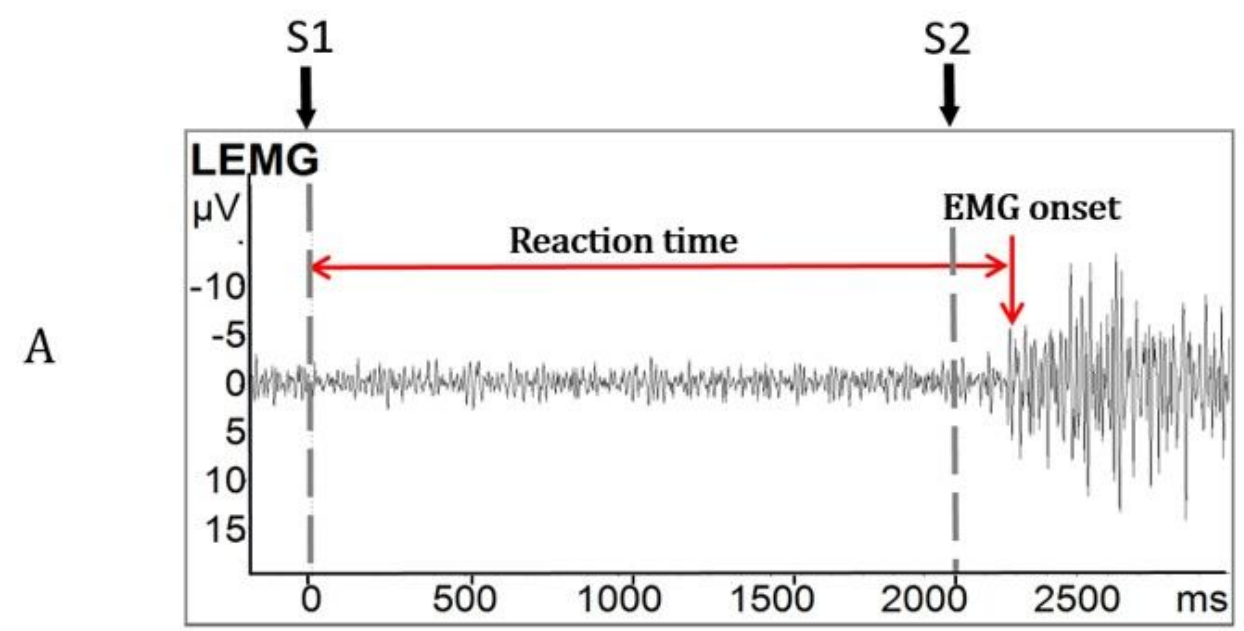

\section{A: EMG reaction time from S1 to EMG onset}

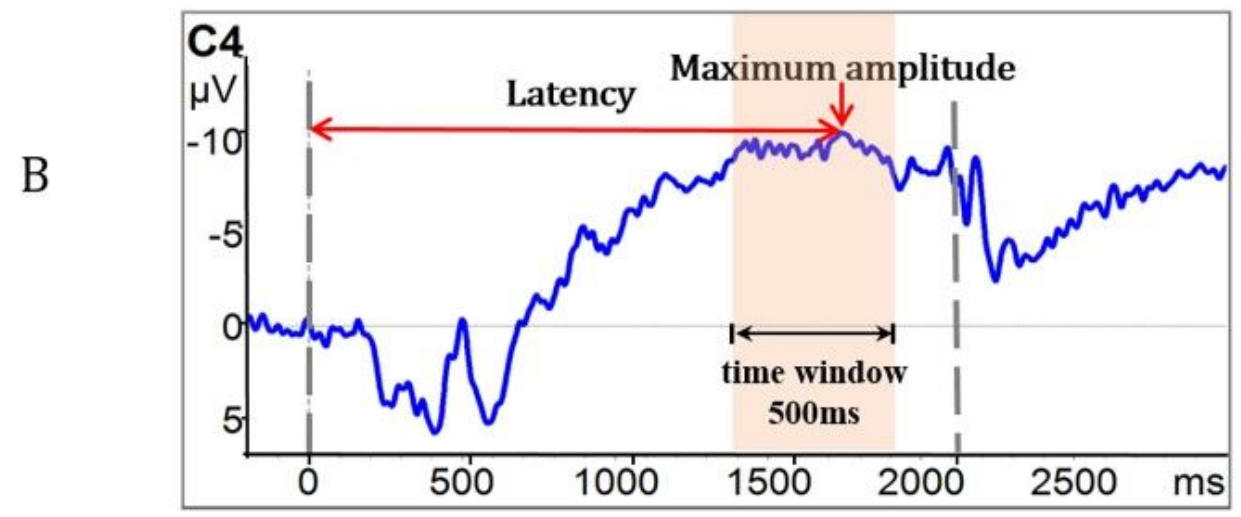

\section{B: Time window for CNV latency and mean amplitude}

Figure 3

Timing window for signal processing data.

Figure 4

Topographic maps of participants in the two groups when executing paretic and non-paretic hand movement tasks. 

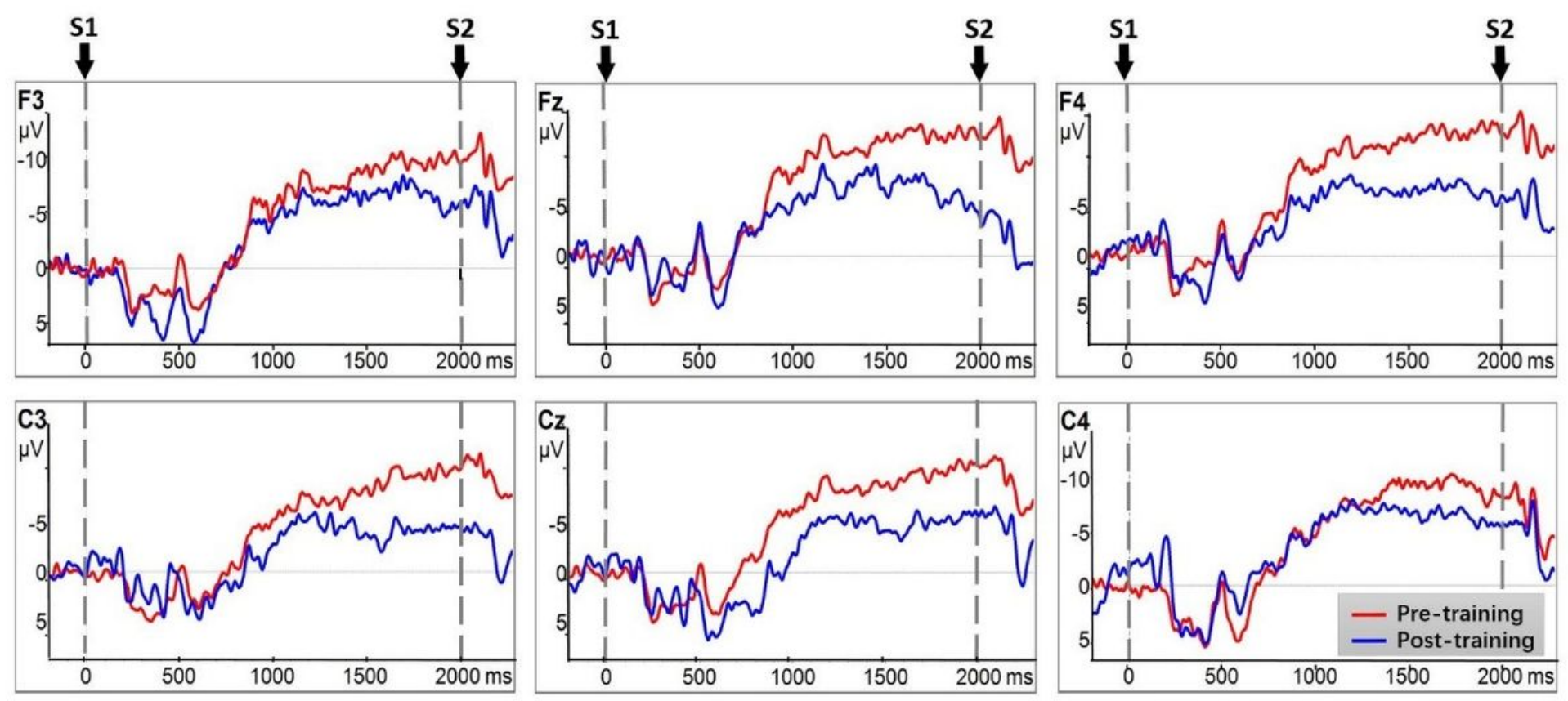

Figure 5

CNV amplitudes of the VR group during paretic hand movement. Negative is plotted upwards. The 200ms epoch prior to S1 onset was the baseline CNV amplitude. SI onset is at $0 \mathrm{~ms}$ point is the $\mathrm{S} 1$ trigger time.

Figure 6

CNV amplitudes of the VR group during non-paretic hand movement.

Figure 7

CNV amplitudes of the control group during paretic hand movement.

\section{Figure 8}

CNV amplitudes of the control group during non-paretic hand movement.

\section{Supplementary Files}

This is a list of supplementary files associated with this preprint. Click to download.

- CONSORT2010Checklist.pdf 